\title{
Pleural plaques in lung cancer screening by low-dose computed tomography: prevalence, association with lung cancer and mortality
}

Mario Silva ${ }^{1,2^{*}}$ D, Nicola Sverzellati ${ }^{1,2}$, Davide Colombi ${ }^{1}$, Gianluca Milanese ${ }^{1,2}$, Carlo La Vecchia ${ }^{3}$, Carlotta Galeone ${ }^{3}$, Alfonso Marchianò ${ }^{4}$ and Ugo Pastorino ${ }^{2}$

\begin{abstract}
Background: To report the prevalence of pleural plaques in a lung cancer screening trial by low-dose computed tomography (LDCT) and to test the association with incidence of lung cancer and mortality.

Methods: The LDCT of 2303 screenees were retrospectively reviewed with the specific aim of describing the prevalence and features of pleural plaques. Self-administered questionnaire was used to assess asbestos exposure. Frequency of lung cancer, lung cancer mortality, and overall mortality were detailed according to presence of pleural findings. Statistical analyses included comparison of mean or median, contingency tables, and Cox model for calculation of hazard ratio (HR) and its 95\% confidence interval (Cl).

Results: Among male screenees, 31/1570 (2\%) showed pleural abnormalities, 128/1570 (8.2\%) disclosed asbestos exposure, 23/31 (74.2\%) subjects with pleural plaques consistently denied exposure to asbestos. There was a trend for higher frequency of lung cancer among subjects with pleural plaques (9.7\% vs $4.2 \%)$. Lung cancer in subjects with pleural plaques was always diagnosed in advanced stage. Subjects with pleural plaques showed HR 5.48 (95\% Cl 1.61-18.70) for mortality from lung cancer.
\end{abstract}

Conclusions: Pleural plaques are a risk factor for lung cancer mortality that can be detected in lung cancer screening by LDCT, also in subjects that are not aware of asbestos exposure.

Trial registration: NCT02837809 - Retrospectively registered July 1, 2016 - Enrolment of first participant September 2005.

Keywords: Lung cancer screening, Pleural abnormalities, Asbestos exposure, Pleural plaques, Self-disclosure of asbestos exposure, Post-test refinement of individual risk

\footnotetext{
* Correspondence: mario.silva@unipr.it

${ }^{1}$ Department of Medicine and Surgery (DiMeC), Section of Radiology, Unit of

Surgical Sciences, University of Parma, Padiglione Barbieri, Via Gramsci 14,

43126 Parma, Italy

${ }^{2}$ Department of Thoracic Surgery, Fondazione IRCCS Istituto Nazionale dei

Tumori, Milan, Italy

Full list of author information is available at the end of the article
} 


\section{Background}

Lung cancer screening by low-dose computed tomography (LDCT) has the main purpose of reducing lung cancer mortality in subjects that have several risk factors contributing to increased risk of lung cancer [1]. Asbestos exposure is a well-known risk factor for thoracic malignancies and non-malignant respiratory diseases [2-5], hence, the majority of lung cancer risk models include exposure to asbestos for pre-test calculation of lung cancer risk [3, 6, 7]. However, there is limited reliability on reference methods for the assessment of life-time exposure from occupational and, notably, non-occupational inhalation.

Signs of asbestos exposure can be detected by LDCT, with variable degrees of accuracy. Pleural plaques are among the chest abnormalities significantly related with exposure to asbestos $[8,9]$. In the setting of professional exposure to asbestos, several studies reported the association between reduced survival and asbestos-related LDCT signs, including pleural plaques $[4,10]$. The detection of pleural plaques and their prognostic value in non-occupational setting has not been investigated, yet. Lung cancer screening trials by LDCT provide the unique opportunity of investigating pleural plaques out of the specific setting of professional surveillance.

The aim of this study was to describe the prevalence of pleural plaques in a population of lung cancer screening participants, and to test their relation with incidence of lung cancer and mortality.

\section{Methods}

The Institutional Review Board approved the Multicenter Italian Lung Detection (MILD) protocol, and written informed consent was obtained from all participants. Informed consent included retrospective evaluation of MILD data, as performed in this study. Therefore, specific consent was waived for this study. Eligibility criteria were as follows: age 50-75 years, current or former (having quit $<10$ years before enrollment) smokers with a tobacco burden $\geq 20$ pack-years, and no history of cancer in the 5 years before enrollment. Details on screening strategy and lung cancer specific mortality rates of the MILD trial are published elsewhere [11]. In brief, the MILD trial enrolled a total of 4099 participants from September 2005 to January 2011. These subjects were randomly assigned to two groups: the early detection group (2376 participants) received a LDCT either every 12 months (1190 participants) or every 24 months (1186 participants), and the control group (1723 participants) received only primary prevention program with pulmonary function test and blood sample collection.

This investigation aimed to evaluate pleural abnormalities on baseline LDCT, and was thus based on the early detection group only. Baseline LDCT was available for 2303 out of 2376 participants (1570 men and 733 women; median age 60 years, interquartile range - IQR 55 to 65 years). These subjects were evaluated for pleural abnormalities. The MILD protocol included selfadministered questionnaire about medical history (i.e. oncologic history, cardio-vascular and respiratory anamnesis, metabolic diseases, and family history of lung cancer), smoking history (i.e. cigarette smokers were differentiated from other smokers, duration of smoking, amount of daily cigarette, pack-years, and Fagerstrom test) [12], exposure to asbestos, respiratory symptoms, subjective awareness of lung cancer risk related to smoking, and willing to quitting smoking [13]. Also, height and weight were recorded and body mass index (BMI) was calculated.

\section{Visual assessment of pleural plaques on LDCT LDCT technique}

LDCT was performed with a 16-detector CT scanner (Somatom Sensation; Siemens Healthcare, Forchheim, Germany) without contrast agent, and with standardized low dose acquisition protocol: potential $120 \mathrm{kVp}$, current 30 effective mAs, collimation $0.75 \mathrm{~mm}$, rotation time $0.5 \mathrm{~s}$, and pitch 1.5. For each LDCT examination, the reconstruction protocol was as follows: slice thickness $1 \mathrm{~mm}$, slice increment $1 \mathrm{~mm}$, and kernel b50. For this study, the reconstructed images were stored to a dedicated workstation for diagnostic work-up (Osirix, Pixmeo SARL, Bernex, Switzerland); window width (WW) and window level (WL) were optimized for assessment of pleura (soft tissue setting: WW $350 \mathrm{HU}$ and WL $50 \mathrm{HU}$ ) and lung parenchyma (lung setting: WW 1600 and WL -600 HU).

\section{Pleural findings}

LDCTs were reviewed by two radiologists for the detection of pleural abnormalities (both radiologists had threeyear experience in lung cancer screening LDCT: 1201 LDCTs read by MS and 1102 LDCTs read by DC). A senior radiologist (NS, with 10-year experience in lung cancer screening LDCT) reviewed LDCT of all subjects with pleural abnormalities and selected those evocative of asbestos exposure, as described thereafter. Pleural plaques and diffuse pleural thickening were deemed findings evocative of asbestos exposure [8, 9]. In particular, pleural plaques were described as solid thickening of pleural surface with clear-cut edges, with or without calcification [14, 15] (Fig. 1). Diffuse pleural thickening was described as single continuous pleural thickening thicker than $3 \mathrm{~mm}$, larger than $5 \mathrm{~cm}$ on axial plane, and longer than $8 \mathrm{~cm}$ in z-axis $[8,14]$. From now on, all pleural abnormalities evocative of asbestos exposure will be referred as pleural plaques. All pleural plaques were visually scored according to a pre-formatted score sheet, adapted from the literature [14-17], namely: a) distribution according to 


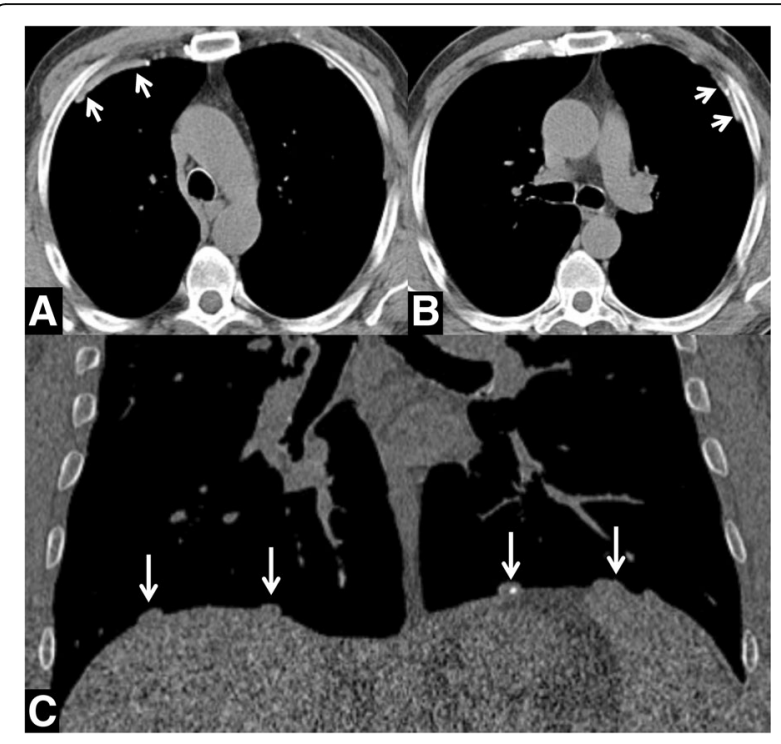

Fig. 1 a-c Pleural plaques. a-b - Transverse CT image of the chest shows smooth solid thickening of pleural surface with clear-cut edge. c - Coronal reconstruction of the chest shows smooth solid thickening of pleural surface above the diaphragm, with scant calcification

side (unilateral or bilateral) and pleural anatomical compartment (subcostal, para-mediastinal, diaphragmatic, and fissural); b) presence of calcifications; c) aspect was described as smooth or nodular; d) cumulative extent was classified into 4 groups: smaller than $1 \mathrm{~cm}$, between $1 \mathrm{~cm}$ and $25 \%$ of pleural surface, $25 \%-50 \%$ of pleural surface, and $>50 \%$ of pleural surface; e) thickness was classified into 4 groups: $<2 \mathrm{~mm}, 2-5 \mathrm{~mm}, 5-$ $10 \mathrm{~mm}$, and $>10 \mathrm{~mm}$.

\section{Self-reported asbestos exposure}

The self-administered questionnaire included disclosure of asbestos exposure. It was administered at the recruitment appointment and at any following LDCT appointment. Subjects with consistent positive disclosure about asbestos exposure over the consecutive questionnaires were deemed exposed. Conversely, non-exposed subjects were those with consistent negative disclosure or inconsistent disclosures along the consecutive questionnaires (e.g. positive disclosure at baseline questionnaire and negative disclosure at follow-up, or vice versa). The self-reported asbestos exposure was compared with the presence of pleural plaques to investigate awareness of exposure.

\section{Frequency of lung cancer and assessment of cause of mortality}

Lung cancers were recorded from the surgical registry, in particular, histology and stage were reported [18]. Mortality from lung cancer and mortality from any cause were retrieved from the National Registry Office database. Data were updated to April 2016.

\section{Statistical analysis}

Statistical analysis was selectively performed on male population to avoid multivariate model bias from genderrelated variables (e.g. height, weight, and BMI) related to the odd distribution of pleural plaques between genders. The continuous variables were given as median with their interquartile range (IQR) and were analyzed using a twosided Student's t-test, if data were normally distributed (based on the Shapiro-Wilk statistic), or a two-sided Wilcoxon rank-sum test, if otherwise. The categorical variables were given as numbers and percentages and were analyzed using the contingency table analysis with the Chi-square or Fisher's test, as appropriate.

The Cox model adjusted for age, BMI, pack-years, and self-disclosure of asbestos exposure was used to calculate the hazard ratio (HR) and the corresponding 95\% confidence intervals (CI) for lung cancer incidence, lung cancer mortality, mortality from causes different from lung cancer, and overall mortality. All tests were twosided and a $p$-value $<0.05$ was considered as statistically significant. All statistical analyses were performed with MedCalc 12 (MedCalc Software, Belgium).

\section{Results}

\section{Visual assessment of pleural plaques on LDCT}

Pleural plaques were reported in 33/2303 subjects (1.4\%), in particular $31 / 1570$ men $(2.0 \%)$ and $2 / 733$ women $(0.3 \%) \quad(p<0.001)$. Demographics, biometrics, smoking history, and self-disclosure of asbestos exposure of male screenees were reported in Table 1, according to presence of pleural plaques. Men with pleural plaques were older $(p=0.005)$, with similar biometrics and smoking history compared to men without pleural plaques. The geographic area of screenee provenance was summarized in Fig. 2 and the distribution of pleural plaques was detailed as the ratio of screenees from each area of provenance. LDCT features of pleural plaques were summarized in Table 2, notably pleural effusion was reported in 1/31 men (3.2\%) and it was not associated with lung cancer or mesothelioma.

\section{Self-reported asbestos exposure}

Asbestos exposure was consistently self-reported by $128 /$ 1570 men (8.2\%), whereas 68/1570 (4.3\%) provided inconsistent disclosure, and 1374/1570 (87.5\%) consistently denied asbestos exposure. The consistent selfreported exposure to asbestos was significantly related to presence of pleural plaques $(p=0.003$; Table 1$)$, however the majority of men with pleural plaques consistently denied exposure to asbestos (23/31, 74.2\%).

\section{Frequency of lung cancer and cause of mortality}

Sixty-eight lung cancers were diagnosed in 1570 men over 14,533.3 person-years (467/100,000 person-years), 
Table 1 Demographics, biometrics, smoking history, and self-disclosure of asbestos exposure in the overall male population and according to the presence of pleural plaques

\begin{tabular}{|c|c|c|c|c|}
\hline & $\begin{array}{l}\text { All men } \\
(n=1570)\end{array}$ & $\begin{array}{l}\text { Men with pleural } \\
\text { plaques }(n=31)\end{array}$ & $\begin{array}{l}\text { Men without pleural } \\
\text { plaques }(n=1539)\end{array}$ & $p^{a}$ \\
\hline Age [years] & $58[54-62]$ & $62[57-66]$ & $58[54-62]$ & 0.0054 \\
\hline Height [cm] & $174[170-178]$ & 177 [170-180] & $174[170-178]$ & 0.2536 \\
\hline Weight [kg] & 80 [73-88] & 83 [75-92] & 80 [73-88] & 0.101 \\
\hline $\mathrm{BMI}[\mathrm{kg} / \mathrm{m} \wedge 2]$ & 26.4 [24.4-28.7] & $27.2[24.8-30.2]$ & $26.4[24.4-28.7]$ & 0.1949 \\
\hline \multicolumn{5}{|l|}{ Smoking history } \\
\hline Current smoker & $1007(64.1 \%)$ & $19(61.3 \%)$ & $988(64.2 \%)$ & \multirow[t]{2}{*}{$0.7103^{b}$} \\
\hline Former smoker & $563(35.9 \%)$ & $12(38.7 \%)$ & $551(35.8 \%)$ & \\
\hline Duration [years] & 39 [34-43] & $40[36-45]$ & 39 [34-43] & 0.2501 \\
\hline Pack years & $40.0[32.0-54.0]$ & $41.0[35.0-61.5]$ & $40.0[32.0-54.0]$ & 0.6378 \\
\hline Self-disclosure of asbestos exposure & $128(8.2 \%)$ & $8(25.8 \%)$ & $120(7.8 \%)$ & $0.0025^{b}$ \\
\hline
\end{tabular}

\section{${ }^{a}$ Wilcoxon test}

${ }^{\mathrm{b}}$ Fisher exact test

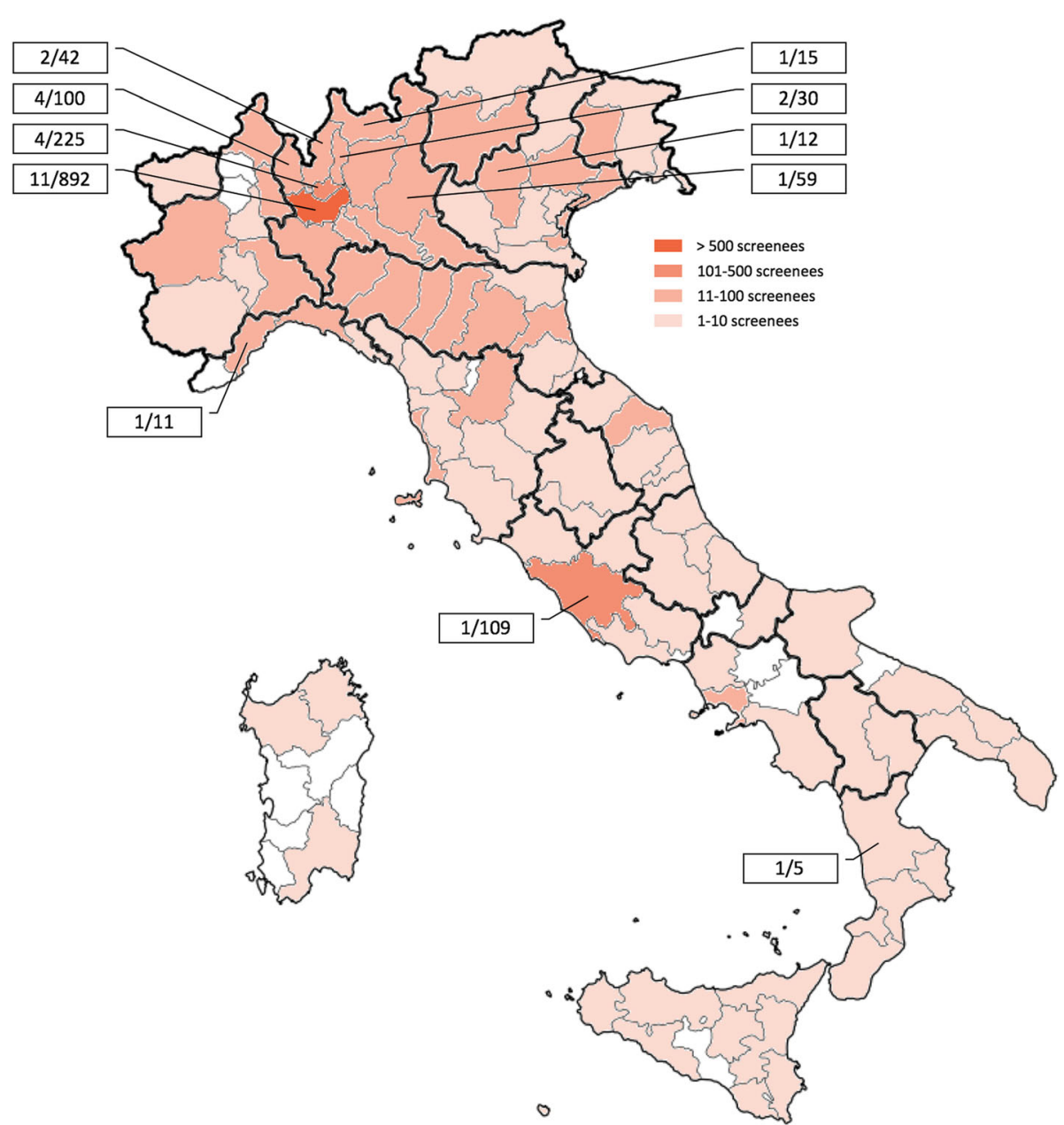

Fig. 2 Geographic area of provenance of screenees are summarized according to numeric categories: 1-10 screenees, 11-100 screenees, 101-500 screenees, and $>500$ screenees. The geographic distribution of pleural plaques is reported as the ratio between the number of screenees with pleural plaques and specific number of screenees from each geographic area (adapted from https://commons.m.wikimedia.org) 
Table 2 LDCT features of pleural plaques detected by LDCT in 31/1570 men

\begin{tabular}{|c|c|}
\hline LDCT features of pleural plaques & $\begin{array}{l}N(\%) \\
31(100 \%)\end{array}$ \\
\hline \multicolumn{2}{|l|}{ Distribution by side: } \\
\hline Bilateral & $28(90 \%)$ \\
\hline Unilateral & $3(10 \%)$ \\
\hline \multicolumn{2}{|l|}{ Distribution by anatomical compartment: } \\
\hline More than one pleural compartment & $21(68 \%)$ \\
\hline Subcostal only & $9(29 \%)$ \\
\hline Para-mediastinal only & $1(3 \%)$ \\
\hline \multicolumn{2}{|l|}{ Extent: } \\
\hline$<1 \mathrm{~cm}$ & $1(3 \%)$ \\
\hline $1 \mathrm{~cm}$ to $25 \%$ & $25(81 \%)$ \\
\hline 25 to $50 \%$ & $4(13 \%)$ \\
\hline$>50 \%$ & $1(3 \%)$ \\
\hline \multicolumn{2}{|l|}{ Thickness: } \\
\hline$<2 \mathrm{~mm}$ & $1(3 \%)$ \\
\hline $2-5 \mathrm{~mm}$ & $7(23 \%)$ \\
\hline $5-10 \mathrm{~mm}$ & 19 (61\%) \\
\hline$>10 \mathrm{~mm}$ & $4(13 \%)$ \\
\hline \multicolumn{2}{|l|}{ Calcification: } \\
\hline Calcified & $21(68 \%)$ \\
\hline Non-calcified & $10(32 \%)$ \\
\hline \multicolumn{2}{|l|}{ Aspect morphology: } \\
\hline Nodular aspect & 19 (61\%) \\
\hline Smooth aspect & $12(39 \%)$ \\
\hline Pleural effusion & $1(3 \%)$ \\
\hline
\end{tabular}

until April 2016 (Table 3). In particular, the frequency of lung cancer was $3 / 31$ in men with pleural plaques $(1079 / 100,000$ person-years) and $65 / 1539$ in men without pleural plaques (456/100,000 person-years), with a non-statistically significant difference between the groups $(p=0.148)$. In particular, all men with pleural plaques showed stage 4 lung cancer. The mortality from lung cancer in men with pleural plaques was statistically higher compared to those without pleural plaques $(p=0.015)$, whereas the mortality from causes other than lung cancer was similar between the two groups.

A single mesothelioma was diagnosed in a subject who did not have pleural plaques nor disclosed asbestos exposure.

Table 4 details the HR for overall or specific mortality and for incidence of lung cancer in subjects with pleural plaques. The HR for overall mortality was higher in men with pleural plaques (HR 2.02), allegedly conditioned by the exceptionally high HR for mortality from lung cancer (HR 5.48). Of note, the HR for lung cancer incidence was slightly but not significantly higher in men with pleural plaques (HR 1.84).

\section{Discussion}

This study reports $2 \%$ prevalence of pleural plaques in male participants of a lung cancer screening trial. Selfdisclosure of asbestos exposure was recorded in $8.2 \%$ of male screenees, however a significant proportion of subjects with pleural plaques were not aware of asbestos exposure. Male screenees with pleural plaques showed a trend for higher risk of lung cancer and significant increase of lung cancer mortality compared to male screenees without pleural plaques.

The relationship between asbestos exposure and lung cancer has been investigated in several studies based on occupational surveillance programs for asbestos workers

Table 3 Comparison of lung cancer frequency, stage, and cause of mortality according to presence pleural plaques. The table includes events from the entire period of lung cancer screening (median follow-up 9.5 years). Notably, pleural plaques were searched on baseline LDCT, therefore detection of pleural plaques either preceded (e.g. event at incidence rounds) or was synchronous (e.g. event at baseline round) to each detailed event

\begin{tabular}{llll}
\hline & $\begin{array}{l}\text { All patients } \\
(n=1570)\end{array}$ & $\begin{array}{l}\text { Men with pleural } \\
\text { plaques }(n=31)\end{array}$ & $\begin{array}{l}\text { Men without pleural } \\
\text { plaques }(n=1539)\end{array}$ \\
\hline Total person-years follow-up & $14,533.3$ & 278.0 & $14,255.3$ \\
Median person-years follow-up & 9.5 & 9.4 & 9.5 \\
$\begin{array}{l}\text { Patients with lung cancer } \\
\text { Lung cancer stage }\end{array}$ & $68(4.3 \%)$ & $3(9.7 \%)$ & $65(4.2 \%)$ \\
$\quad$ I or II & $39(57.4 \%)$ & 0 & $39(60 \%)$ \\
$\quad$ III or IV & $29(42.6 \%)$ & $3(100 \%)$ & $26(40.0)$ \\
Total deaths & $87(5.5 \%)$ & $4(12.9 \%)$ & $83(5.4 \%)$ \\
Lung cancer deaths & $27(1.7 \%)$ & $3(9.7 \%)$ & $24(1.6 \%)$ \\
Deaths from causes other than lung cancer & $60(3.8 \%)$ & $1(3.2 \%)$ & $59(3.8 \%)$ \\
\hline
\end{tabular}

\footnotetext{
${ }^{\mathrm{a}}$ Fisher exact test
} 
Table 4 Hazard ratios $(H R)^{a}$ of overall mortality, lung cancer mortality and lung cancer occurrence among 1570 men according to pleural findings

\begin{tabular}{ll}
\hline & $\begin{array}{l}\text { Pleural findings } \\
\mathrm{HR}^{\mathrm{a}}(95 \% \mathrm{Cl})\end{array}$ \\
\hline Overall mortality $(\mathrm{N}=87)$ & $2.02(0.73-5.56)$ \\
Mortality from lung cancer $(N=27)$ & $5.48(1.61-18.70)$ \\
Mortality from other causes than & $0.70(0.10-5.10)$ \\
lung cancer $(N=60)$ & \\
Lung cancer incidence $(N=68)$ & $1.84(0.57-5.93)$ \\
\hline
\end{tabular}

${ }^{a}$ Estimated from Cox model adjusted for age, BMI, pack-years, self-disclosure of asbestos exposure

[19-22]. Current or recent occupational exposure to asbestos is a known risk factor for lung cancer with a reported two-fold increase of lung cancer risk among smokers [7]. Mastrangelo et al. reported progressive decrease of relative risk of lung cancer after 15-20 years since last exposure to asbestos [22], comparable to smoking cessation. Nevertheless, increased risk of thoracic malignancy has been demonstrated also for extremely low exposure, such as non-occupational and environmental exposure [23, 24]. Accordingly, several lung cancer risk models for selection of lung cancer screening population include asbestos exposure among risk factors, even in case of non-occupational exposure $[3,7]$. Non-occupational exposure is likely to become more relevant in industrialized countries were asbestos ban was issued more than 20 years ago [10]. The assessment of non-occupational exposure to asbestos is extremely challenging, indeed more controversial that the assessment of occupational exposure [25]. Selfadministered questionnaires have been employed to collect data on asbestos exposure in participants of lung cancer screening trials, despite this assessment demands specialized operators for thorough investigation of potential pollutant [26]. Our data showed that signs of asbestos exposure such pleural plaques were associated with increased risk of lung cancer mortality, including subjects who were not aware of occupational exposure. The increased risk was independent from tobacco burden. We encourage specific investigation of asbestos exposure (both occupational and non-occupational) for selection of subjects for lung cancer screening, even when tobacco burden does not meet the reference level for recruitment in such screening.

Previous lung cancer screening trials reported prevalence of asbestos-exposure ranging 5.6-7.3\% [27, 28]. In our study, similar results were obtained by self-disclosure of asbestos exposure (overall self-disclosed exposure 6.5\% - data not reported in results), however almost 75\% of male screenees with pleural plaques did not disclose the exposure. This observation confirms that self-disclosure of asbestos exposure is not reliable, as it was reported in the literature [29]. Ledda et al. reported a significant increase of pleural plaques prevalence among subjects in an area with high environmental concentration of fibrous amphibole [30]. Of note, the CT features of pleural plaques reported by Ledda in environmentally exposed subjects were similar to those reported in our lung cancer screening population, in particular the majority of subjects showed plaque extent between $1 \mathrm{~cm}$ and $25 \%$ of pleural surface. Pleural plaques in subjects that are not aware of exposure to asbestos could be associated with non-occupational and environmental exposure, and this should be particularly emphasized in high-risk subjects that undergo lung cancer screening by LDCT.

Bach et al. showed increase of lung cancer risk in association to asbestos exposure in participants of lung cancer screening trials [31]. In our study, screenees with pleural plaques showed higher risk of lung cancer mortality compared to the remainder screenees. We underline that screenees with pleural plaques were diagnosed with advanced stage lung cancer. Hence, we suggest that increased mortality could have been driven by a more aggressive pattern of lung cancer in this group of screenees. Again, it is apparent that assessment of pleural plaques in lung cancer screening participant contributes to the detection of subjects with minor or unknown exposure. For this purpose, the objective assessment of pleural abnormalities could be regarded as potential marker of increased risk of lung cancer associated to asbestos exposure, and should be investigated for post-test refinement of subjective risk of lung cancer. Furthermore, from a demographic point of view, detection of pleural plaques in screenees that are not aware of the exposure should prompt active investigation of environmental exposure to asbestos in specific areas.

Among occupationally exposed subjects, the correlation between pleural plaques and lung cancer appears to be lower compared to the relative risk associated with asbestosis [10]. Both asbestosis and pleural plaques can be seen on LDCT, however pleural plaques are more specific than asbestosis findings in the absence of known exposure to asbestos. Vehmas et al. reported significant increase of lung cancer death in asbestos workers with pleural plaques [4]. This report in workers exposed to asbestos is confirmed by our data in the setting of lung cancer screening trial. Screenees with pleural plaques showed a trend for increased incidence of lung cancer, noteworthy the clinical evolution of lung cancer in these subjects was extremely aggressive. Lung cancer in subjects with pleural plaques were all diagnosed in stage 4 and, thus, lung cancer mortality was significantly higher compared to screenees with cancer diagnosis but without pleural plaques, suggesting that mortality from lung cancer in subjects with pleural plaques could be influenced by more factors rather than the simple incidence of lung cancer (e.g. aggressive biology). Risk models for post-test risk refinement 
are mostly based on the presence and characteristics of nodule and have been validated [32-34]. Looking forward to screening implementation, post-test risk model enrichment is fostered including other non-nodular LDCT findings such as emphysema and other interstitial lung abnormalities. Pleural plaques should be considered as additional non-nodular finding for post-test risk model.

This study has some limitations. Asbestos exposure was investigated by prospectively self-administered questionnaire, which is known to be inconsistent for assessment of asbestos exposure. However, self-administered questionnaire is the affordable option for implementation in lung cancer screening by LDCT. Moreover, asbestos exposure was not further investigated in subjects with pleural plaques and unaware of asbestos exposure. This could be regarded as a limitation because the specific description of exposure hazard in this subgroup could have provided information to improve anamnestic collection of this specific pollutant. Moreover, the asbestos exposure was investigated with a binary question (e.g. yes or no), without specific description of exposure duration and intensity. This limitation was driven by the management and financial plan within lung cancer screening trial with a large population of screenees. We foster future studies testing the correlation between detailed descriptors of asbestos exposure and lung cancer outcomes within lung cancer screening programs. Finally, parenchymal signs of asbestos exposure were not investigated, despite they are associated with risk of lung cancer. Notably, parenchymal signs were not scored because they are not specific for asbestos exposure when the exposure is not formally confirmed. It would be ideal that reliable information about asbestos exposure in lung cancer screening allow specific investigation of interstitial lung abnormalities related with the exposure, since they are significantly related with the risk of lung cancer [10].

\section{Conclusions}

In conclusion, pleural plaques can be detected in a sizeable proportion of subjects undergoing lung cancer screening by LDCT. Screenees with pleural plaques show increased risk of lung cancer mortality, therefore pleural plaques could be considered in risk models for post-test refinement of subjective risk.

\section{Abbreviations}

BMl: Body mass index; Cl: Confidence interval; HR: Hazard ratio; IQR: Interquartile range; LDCT: Low-dose computed tomography; WL: Window level; WW: Window width

\section{Acknowledgements}

The authors would like to thank all the MILD participants and their families. Moreover, the authors wish to thank Claudio Jacomelli and Claudio Citterio for data management and the MILD staff (Elena Bertocchi, Annamaria Calanca, Carolina Ninni, Stefano Sestini, Paola Suatoni). The Authors thank Dr. Francesca Marezza (University of Parma) for her contribution in the field of medico-legal implication of asbestos exposure.

\section{Funding}

The MILD project was supported by grants from the Italian Ministry of Health (RF 2004), the Italian Association for Cancer Research (AIRC 2004 IG 1227 and AIRC 5xmille IG 12162), and the Fondazione Cariplo (2004-1560).

\section{Availability of data and materials}

The data that support the findings of this study are available from Fondazione IRCCS Istituto Nazionale dei Tumori di Milano but restrictions apply to the availability of these data, which were used under license for the current study, and so are not publicly available. Data are however available from the authors upon reasonable request and with permission of Fondazione IRCCS Istituto Nazionale dei Tumori di Milano.

\section{Authors' contributions}

Study concepts and design: NS, MS, DC, UP, CLV. Literature research: MS, NS, DC, GM, AM. Data collection: MS, DC, NS, GM, CG. Data Analysis: MS, NS, UP, CLV, CG. Statistical analysis: CG, CLV. Manuscript preparation: MS, NS, UP. Manuscript editing: DC, GM, CLV, CG, AM. All authors have read and approved the final version of this manuscript.

\section{Ethics approval and consent to participate}

The Institutional Review Board of the Fondazione IRCCS Istituto Nazionale dei Tumori di Milano approved the Multicenter Italian Lung Detection (MILD) protocol, and written informed consent was obtained from all participants. Informed consent included retrospective evaluation of MILD data, as performed in this study. Therefore, specific consent was waived for this study.

\section{Consent for publication}

Not applicable

\section{Competing interests}

The authors declare that they have no competing interests..

\section{Publisher's Note}

Springer Nature remains neutral with regard to jurisdictional claims in published maps and institutional affiliations.

\section{Author details}

${ }^{1}$ Department of Medicine and Surgery (DiMeC), Section of Radiology, Unit of Surgical Sciences, University of Parma, Padiglione Barbieri, Via Gramsci 14, 43126 Parma, Italy. ${ }^{2}$ Department of Thoracic Surgery, Fondazione IRCCS Istituto Nazionale dei Tumori, Milan, Italy. ${ }^{3}$ Department of Clinical Sciences and Community Health, University of Milan, Milan, Italy. ${ }^{4}$ Department of Radiology and Radiotherapy, Fondazione IRCCS Istituto Nazionale dei Tumori, Milan, Italy.

Received: 11 January 2017 Accepted: 16 November 2017

Published online: 25 November 2017

References

1. Field JK, Devaraj A, Duffy SW, Baldwin DR. CT screening for lung cancer: is the evidence strong enough? Lung Cancer. 2016;91:29-35.

2. Hammond EC, Selikoff IJ, Seidman H. Asbestos exposure, cigarette smoking and death rates. Ann N Y Acad Sci. 1979;330:473-90.

3. Cassidy A, Myles JP, van Tongeren M, Page RD, Liloglou T, Duffy SW, Field JK. The LLP risk model: an individual risk prediction model for lung cancer. $\mathrm{Br}$ J Cancer. 2008:98(2):270-6.

4. Vehmas T, Oksa P, Kivisaari L. Lung and pleural $C T$ signs predict deaths: 10-year follow-up after lung cancer screening of asbestos-exposed workers. Int Arch Occup Environ Health. 2012;85(2):207-13.

5. Lopatin S, Tsay JC, Addrizzo-Harris D, Munger JS, Pass H, Rom WN. Reduced lung function in smokers in a lung cancer screening cohort with asbestos exposure and pleural plaques. Am J Ind Med. 2016;59(3):178-85.

6. Bach PB, Kattan MW, Thornquist MD, Kris MG, Tate RC, Barnett MJ, Hsieh LJ, Begg CB. Variations in lung cancer risk among smokers. J Natl Cancer Inst. 2003:95(6):470-8.

7. Spitz MR, Hong WK, Amos Cl, Wu X, Schabath MB, Dong Q, Shete S, Etzel CJ. A risk model for prediction of lung cancer. J Natl Cancer Inst. 2007:99(9):715-26.

8. Gevenois PA, de Maertelaer V, Madani A, Winant C, Sergent G, De Vuyst P. Asbestosis, pleural plaques and diffuse pleural thickening: three distinct benign responses to asbestos exposure. Eur Respir J. 1998;11(5):1021-7. 
9. Tiitola M, Kivisaari L, Zitting A, Huuskonen MS, Kaleva S, Tossavainen A, Vehmas T. Computed tomography of asbestos-related pleural abnormalities. Int Arch Occup Environ Health. 2002;75(4):224-8.

10. Mastrangelo G, Marangi G, Ballarin MN, Fadda E, Scoizzato L, Fedeli U, Marchiori L, Valentini F. Lung cancer risk in past asbestos workers a few decades after exposure cessation and prospects for screening. Arch Environ Occup Health. 2016;71(4):237-44.

11. Sverzellati N, Silva M, Calareso G, Galeone C, Marchiano A, Sestini S, et al. Low-dose computed tomography for lung cancer screening: comparison of performance between annual and biennial screen. Eur Radiol. 2016;26(11): 3821-9.

12. Fagerstrom KO. Measuring degree of physical dependence to tobacco smoking with reference to individualization of treatment. Addict Behav. 1978;3(3-4):235-41.

13. Pastorino U, Boffi R, Marchiano A, Sestini S, Munarini E, Calareso G, Boeri M, Pelosi G, Sozzi G, Silva M, et al. Stopping smoking reduces mortality in low-dose computed tomography screening participants. J Thoracic Oncol. 2016;11(5):693-9.

14. Remy-Jardin M, Sobaszek A, Duhamel A, Mastora I, Zanetti C, Remy J. Asbestos-related pleuropulmonary diseases: evaluation with low-dose four-detector row spiral CT. Radiology. 2004;233(1):182-90.

15. Meirelles GS, Kavakama Jl, Jasinowodolinski D, Nery LE, Terra-Filho M, Rodrigues RT, Neder JA, Napolis LM, Bagatin E, D'Ippolito G, et al. Pleural plaques in asbestos-exposed workers: reproducibility of a new highresolution $C T$ visual semiquantitative measurement method. J Thorac Imaging. 2006;21(1):8-13.

16. Paris C, Thierry S, Brochard P, Letourneux M, Schorle E, Stoufflet A, Ameille J, Conso F, Pairon JC, National AM. Pleural plaques and asbestosis: dose- and time-response relationships based on HRCT data. Eur Respir J. 2009;34(1):72-9.

17. Hansell DM: Imaging of diseases of the chest, 5th ed. / David M. Hansell ... [et al.]. edn. [Edinburgh?]: Mosby; 2010.

18. Goldstraw P, Crowley J, Chansky K, Giroux DJ, Groome PA, Rami-Porta R, Postmus PE, Rusch V, Sobin L, International Association for the Study of Lung Cancer International Staging C, et al. The IASLC lung cancer staging project: proposals for the revision of the TNM stage groupings in the forthcoming (seventh) edition of the TNM classification of malignant tumours. J thoracic Oncol. 2007;2(8):706-14.

19. Brims FJ, Murray CP, de Klerk N, Alfonso H, Reid A, Manners D, Wong PM, Teh J, Olsen N, Mina R, et al. Ultra-low-dose chest computer tomography screening of an asbestos-exposed population in Western Australia. Am J Respir Crit Care Med. 2015;191(1):113-6.

20. Murray CP, Wong PM, Teh J, de Klerk N, Rosenow T, Alfonso H, et al. Ultra low dose CT screen-detected non-malignant incidental findings in the Western Australian Asbestos Review Programme. Respirology. 2016;21(8): 1419-24.

21. Markowitz SB, Levin SM, Miller A, Morabia A. Asbestos, asbestosis, smoking, and lung cancer. New findings from the north American insulator cohort. Am J Respir Crit Care Med. 2013;188(1):90-6.

22. Mastrangelo G, Ballarin MN, Bellini E, Bicciato F, Zannol F, Gioffre F, Zedde A, Tessadri G, Fedeli U, Valentini F, et al. Asbestos exposure and benign asbestos diseases in 772 formerly exposed workers: dose-response relationships. Am J Ind Med. 2009;52(8):596-602.

23. Baumann F, Buck BJ, Metcalf RV, McLaurin BT, Merkler DJ, Carbone M. The presence of asbestos in the natural environment is likely related to Mesothelioma in young individuals and women from southern Nevada. J thoracic Oncol. 2015;10(5):731-7.

24. Ferrante D, Mirabelli D, Tunesi S, Terracini B, Magnani C. Pleural mesothelioma and occupational and non-occupational asbestos exposure: a case-control study with quantitative risk assessment. Occup Environ Med. 2016;73(3):147-53.

25. Wheeler DC, Burstyn I, Vermeulen R, Yu K, Shortreed SM, Pronk A, Stewart PA, Colt JS, Baris D, Karagas MR, et al. Inside the black box: starting to uncover the underlying decision rules used in a one-by-one expert assessment of occupational exposure in case-control studies. Occup Environ Med. 2013;70(3):203-10.

26. Cox CW, Rose CS, Lynch DA. State of the art: imaging of occupational lung disease. Radiology. 2014;270(3):681-96.

27. Tockman MS. Clinical detection of lung cancer progression markers. J Cell Biochem Suppl. 1996;25:177-84.

28. Melamed MR. Lung cancer screening results in the National Cancer Institute new York study. Cancer. 2000;89(11 Suppl):2356-62.
29. Niklinski J, Niklinska W, Chyczewska E, Laudanski J, Naumnik W, Chyczewski L, Pluygers E. The epidemiology of asbestos-related diseases. Lung Cancer. 2004;45(Suppl 1):S7-S15.

30. Ledda C, Pomara C, Bracci M, Mangano D, Ricceri V, Musumeci A, Ferrante M, Musumeci G, Loreto C, Fenga C, et al. Natural carcinogenic fiber and pleural plaques assessment in a general population: a cross-sectional study. Environ Res. 2016;150:23-9.

31. Bach PB, Elkin EB, Pastorino U, Kattan MW, Mushlin Al, Begg CB, Parkin DM. Benchmarking lung cancer mortality rates in current and former smokers. Chest. 2004;126(6):1742-9.

32. Wille MM, Thomsen LH, Petersen J, de Bruijne M, Dirksen A, Pedersen JH, Shaker SB. Visual assessment of early emphysema and interstitial abnormalities on CT is useful in lung cancer risk analysis. Eur Radiol. 2016; 26(2):487-94.

33. Sverzellati N, Randi G, Spagnolo P, Marchiano A, Silva M, Kuhnigk JM, La Vecchia C, Zompatori M, Pastorino U. Increased mean lung density: another independent predictor of lung cancer? Eur J Radiol. 2013;82(8):1325-31.

34. Mascalchi M, Picozzi G, Falchini M, Vella A, Diciotti S, Carrozzi L, et al. Initial LDCT appearance of incident lung cancers in the ITALUNG trial. Eur J Radiol. 2014;83(11):2080-6.

\section{Submit your next manuscript to BioMed Central and we will help you at every step:}

- We accept pre-submission inquiries

- Our selector tool helps you to find the most relevant journal

- We provide round the clock customer support

- Convenient online submission

- Thorough peer review

- Inclusion in PubMed and all major indexing services

- Maximum visibility for your research

Submit your manuscript at www.biomedcentral.com/submit
C) Biomed Central 